Journal of

Applied

Crystallography

ISSN 0021-8898

Editor: Gernot Kostorz

Polarization-dependence of anomalous scattering in brominated DNA and RNA molecules, and importance of crystal orientation in single- and multiple-wavelength anomalous diffraction phasing

R. Sanishvili, C. Besnard, F. Camus, M. Fleurant, P. Pattison, G. Bricogne and M. Schiltz

Copyright $\odot$ International Union of Crystallography

Author(s) of this paper may load this reprint on their own web site provided that this cover page is retained. Republication of this article or its storage in electronic databases or the like is not permitted without prior permission in writing from the IUCr. 
Journal of

Applied

Crystallography

ISSN 0021-8898

Received 20 December 2006

Accepted 28 March 2007
(C) 2007 International Union of Crystallography

Printed in Singapore - all rights reserved

\section{Polarization-dependence of anomalous scattering in brominated DNA and RNA molecules, and importance of crystal orientation in single- and multiple-wavelength anomalous diffraction phasing}

\author{
R. Sanishvili, ${ }^{a} \ddagger$ C. Besnard,${ }^{b} \neq$ F. Camus, ${ }^{b}$ M. Fleurant,,${ }^{b}$ P. Pattison, ${ }^{b, c}$ G. Bricogne ${ }^{d}$ \\ and M. Schiltz ${ }^{\mathrm{b}}$ * \\ ${ }^{a}$ GM/CA CAT, Argonne National Laboratory, 9700 South Cass Avenue, Argonne, IL 60439, USA, \\ bécole Polytechnique Fédérale de Lausanne (EPFL), Laboratoire de Cristallographie, $\mathrm{CH}-1015$ \\ Lausanne, Switzerland, 'Swiss-Norwegian Beamlines at the ESRF, 6 rue Jules Horowitz, BP 220, \\ F-38043 Grenoble CEDEX 9, France, and d Global Phasing Ltd, Sheraton House, Castle Park, \\ Cambridge CB3 OAX, UK. Correspondence e-mail: marc.schiltz@epfl.ch
}

\section{Introduction}

Anomalous scattering methods have achieved tremendous successes in the structure determination of proteins and nucleic acids by X-ray diffraction (Ogata, 1998; Hendrickson, 1999). These methods exploit the resonant behaviour of heavy atoms in the macromolecule when the photon energy of the incident X-rays is in the vicinity of one of their absorption edges (Hendrickson, 1991). Resonance effects produce variable phase shifts that lead to the breakdown of Friedel's law, and the resulting intensity differences serve for determining structure-factor phases. Anomalously scattering atoms can be naturally present in the macromolecule (as e.g. in metalloproteins) or else they may have been incorporated either by chemical attachment or via recombinant DNA technologies. The incorporation of selenomethionine into recombinant proteins (Hendrickson et al., 1990) has now become the dominant method of labelling proteins with anomalously scattering atoms. Today, the vast majority of new protein crystal structures are solved by anomalous scattering experiments on selenated samples. The chemical incorporation of brominated nucleobases has become an equally important

¥ These authors contributed equally to this work. technique for the structure determination of nucleic acids (Correll et al., 1997). The resonance effects taking place in the anomalously scattering atoms are expressed in terms of the real and imaginary anomalous scattering factors $f^{\prime}$ and $f^{\prime \prime}$, which are functions of the X-ray photon energy (i.e. of the wavelength of the radiation) and show significant variations in the vicinity of absorption edges. Anomalous scattering methods are therefore chiefly applied at synchrotron radiation sources, where the wavelength is tunable over a large spectral range (Hendrickson, 1999). In order to maximize the phasing power in anomalous scattering methods, X-ray data are collected at one (SAD, single-wavelength anomalous diffraction) or several wavelengths (MAD, multi-wavelength anomalous diffraction) where the anomalous scattering factors $f^{\prime}$ and $f^{\prime \prime}$ reach extremum values (e.g. minimum of $f^{\prime}$ and maximum of $\left.f^{\prime \prime}\right)$. The phasing power generated by anomalous differences is directly proportional to $f^{\prime \prime}$. Thus, in MAD experiments, at least one of the measurements is carried out at the wavelength where $f^{\prime \prime}$ is maximum, and in an optimized SAD experiment this is usually the single wavelength that is selected. Specific amplification of the anomalous signal strengths can be achieved by exploiting the near-edge features in $f^{\prime \prime}$ spectra, which often display a narrow peak: the so-called 'white line'. Thus, the success of a MAD or optimized SAD 
experiment depends critically on the careful choice of the wavelengths (González, 2003). To that purpose, an X-ray absorption spectrum (in fluorescence excitation mode) of the crystal sample is usually recorded prior to the collection of diffraction data. This spectrum can be transformed into $f^{\prime}$ and $f^{\prime \prime}$ spectra which enable the experimenter to select the optimal wavelengths (Evans \& Pettifer, 2001). Devices for recording such absorption spectra are now standard equipment at SAD/ MAD-capable synchrotron beamlines. While the SAD/MAD methods are robust and have been very successful, they still do not always lead to structure solution. They are typically prone to failure when the anomalous signal is too weak. In such situations, even small enhancements of the anomalous signal can make the difference between success and failure of experimental phasing.

The anomalous scattering terms for isolated atoms are scalars but, generally, chemical bonding and the symmetry of the atom's environment induce a directional dependence of $f^{\prime}$ and $f^{\prime \prime}$ on the direction of linear polarization of the X-ray beam. Such anisotropy of anomalous scattering is, however, only significant in the near-edge region, as has been experimentally demonstrated in numerous investigations for salts and small-molecule compounds (Templeton \& Templeton, 1982, 1988, 1995; Kirfel et al., 1991; Dmitrienko, 1983). A comprehensive review of these studies has been presented by Dmitrienko et al. (2005). Anisotropy of anomalous scattering has also been observed for selenated proteins (Hendrickson $e t$ al., 1990; Fanchon \& Hendrickson, 1990; Bricogne et al., 2005), and in metalloproteins (Hendrickson et al., 1988). Since the $\mathrm{X}$-radiation delivered from synchrotron sources is almost linearly polarized, there can be pronounced modulations of $f^{\prime}$ and $f^{\prime \prime}$ as a function of the crystal orientation. In the present communication, we (i) report measurements of the anisotropy of anomalous scattering in brominated nucleotides and (ii) demonstrate that the correct choice of sample orientation can be as important as the correct choice of wavelength for the success of a SAD or MAD experiment, particularly in the case of brominated nucleic acids. Earlier we have suggested that the anomalous scattering signal strength in selenated protein crystals might be improved by a judicious choice of crystal orientation (Bricogne et al., 2005). Here we show that in the case of brominated nucleic acids, choosing the optimal crystal orientation can be crucial for a successful structure determination.

\section{Anisotropy of anomalous scattering in 5-bromouracil}

In a brominated DNA or RNA molecule, the bromine atom is bound to a carbon atom of the nucleobase ring system (Correll et al., 1997). Thus, 5-bromouracil is frequently used as a substitute for uracil or thymine in order to exploit the anomalous scattering effects of bromine since the linear $\mathrm{C}-$ $\mathrm{Br}$ bond can give rise to a strong anisotropy of anomalous scattering at the $\mathrm{Br} K$ edge (Templeton \& Templeton, 1995; Bricogne et al., 2005). Although there have been attempts to include the anisotropy of anomalous scattering in data processing and phasing methods for macromolecular structure determination (Fanchon \& Hendrickson, 1990), these effects are not routinely taken into consideration. This can be partly explained by the fact that relatively little is known about the tensor properties of the scattering factors of covalently bound anomalous scatterers that are normally used in bio-macromolecules. Indeed, the vast majority of experimental studies of anisotropy of anomalous scattering were conducted on inorganic salt and oxide crystals. Here, we have measured a series of polarized absorption spectra of a single crystal of 5-bromouracil in order to completely map the polarization dependence of anomalous scattering in brominated nucleobases. These data may serve as standards for improved modelling of anomalous scattering factors in macromolecular structure research (i.e. by replacing theoretical or experimental isotropic single-atom $f^{\prime}$ and $f^{\prime \prime}$ curves).

\subsection{Experimental}

Crystals of 5-bromouracil were grown by dissolving the commercially available product (Acros Chemicals) in hot water $(353 \mathrm{~K})$, followed by slow cooling of the solution to room temperature. A single crystal of size $100 \times 30 \times 30 \mu \mathrm{m}$ was mounted on a glass fibre and cooled to $100 \mathrm{~K}$ in a nitrogen cryostream for subsequent measurements. All X-ray experiments were carried out at station BM01A of the SwissNorwegian Beamlines (SNBL) at the European Synchrotron Radiation Facility (ESRF) in Grenoble, France. This beamline is installed at a bending magnet and is equipped with a double $\mathrm{Si}(111)$ crystal monochromator and focusing mirrors. The endstation is a KM6-CH six-circle kappa diffractometer manufactured by Oxford Diffraction Ltd, equipped with a large-size Onyx CCD area detector. Previous measurements have established that the degree of linear polarization in the horizontal plane (i.e. the plane defined by the electron orbit in the synchrotron storage ring) of the X-radiation is better than 0.96 (Birkedal, 2000). A first diffraction data set was recorded in order to assess the crystal quality and to determine an orientation matrix. Polarized absorption spectra were then measured in fluorescence excitation mode using a Ce:YAP scintillation counter (Bede PLC EDRc X-ray detector) mounted in the horizontal plane and perpendicular to the incident beam direction in order to minimize background contributions from elastic scattering processes. For the recording of absorption spectra around the $\mathrm{Br} K$ edge (theoretical free atom value: $E=13474 \mathrm{eV}, \lambda=0.9202 \AA$ ) the following layout was used: (i) energy range $13300-13440 \mathrm{eV}$ : steps of $2 \mathrm{eV}$; (ii) energy range $13440-13520 \mathrm{eV}$ : steps of $0.5 \mathrm{eV}$; (iii) energy range $13520-13700 \mathrm{eV}$ : steps of $2 \mathrm{eV}$; (iv) energy range $13700-13900 \mathrm{eV}$ : steps of $4 \mathrm{eV}$. The counting time at each step was $2 \mathrm{~s}$ and the incident beam was attenuated so as to obtain maximum counts of the order of $10^{6}$.

The sample crystallizes in space group $P 2_{1} / c$ with cell parameters $a=8.663$ (2), $b=6.927$ (2), $c=11.497$ (4) $\AA$ and $\beta=$ $123.92(3)^{\circ}$. There are four symmetry-related molecules of 5bromouracil in the unit cell (Fig. 1), packed with all $\mathrm{C}-\mathrm{Br}$ bonds almost perpendicular to [0 10$]$ and within $20^{\circ}$ parallel to [1 00 0] (Sternglanz \& Bugg, 1975). Absorption spectra were 


\section{research papers}

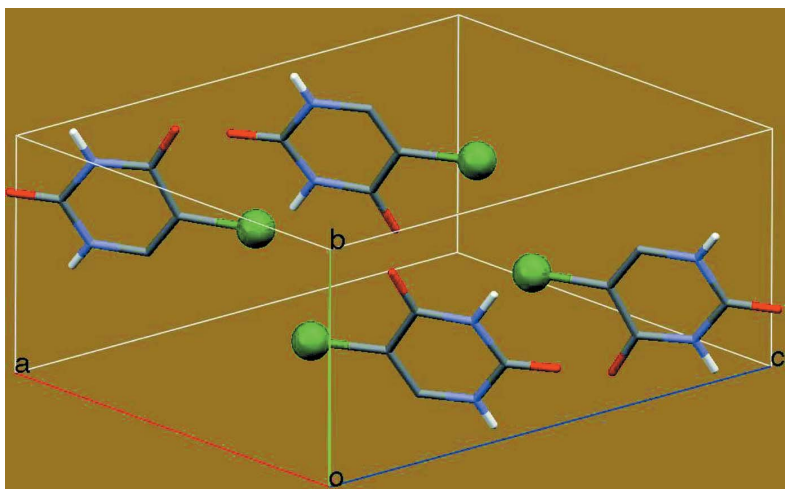

Figure 1

Molecule packing in the unit cell of 5-bromouracil crystals. The $\mathrm{Br}$ atoms are highlighted as green spheres. All $\mathrm{C}-\mathrm{Br}$ bonds are almost perpendicular to [010] and within $20^{\circ}$ to [ 100$]$.

recorded for seven different crystal orientations, for which the polarization direction of the X-radiation was aligned along

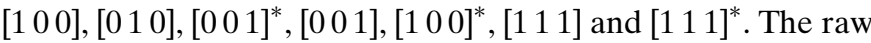
absorption spectra were converted to $f^{\prime}$ and $f^{\prime \prime}$ spectra using the program CHOOCH (Evans \& Pettifer, 2001).

\subsection{Results and discussion}

In the vicinity of the $\mathrm{Br} K$ edge, polarized absorption spectra of 5-bromouracil exhibit a pronounced linear dichroism (Fig. 2). In order to obtain information about the

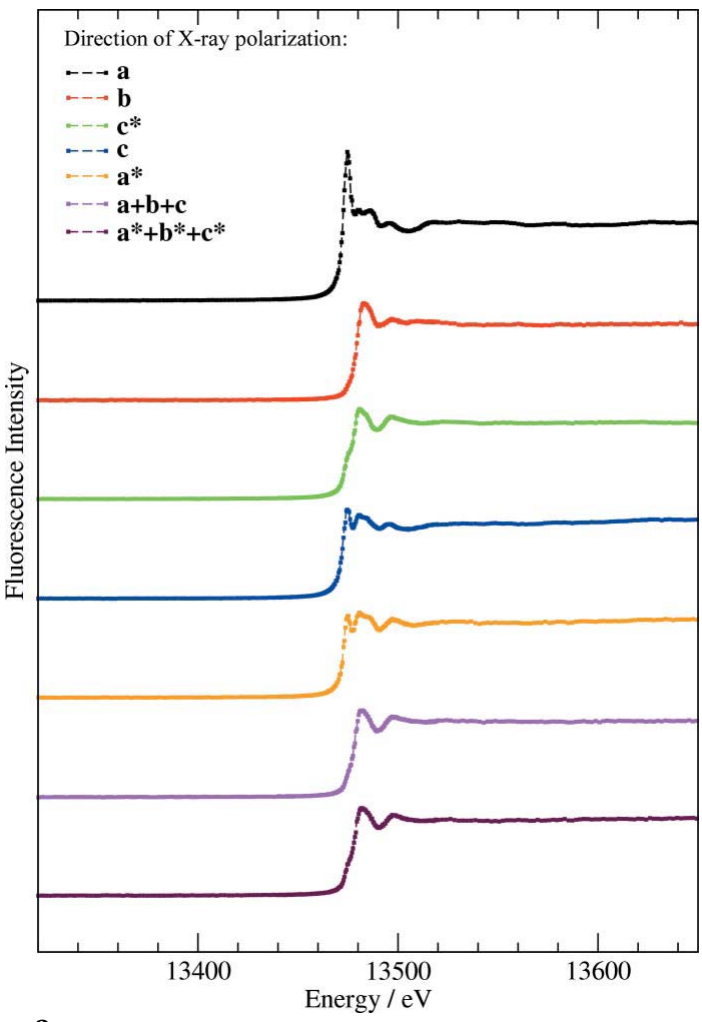

Figure 2

Polarized absorption spectra at the $\mathrm{Br} K$ edge for seven orientations of 5-bromouracil crystals. The plots show the fluorescence intensity yields after normalization. Thus, all spectra are on the same scale, but are arbitrarily shifted on the vertical axis for clarity.

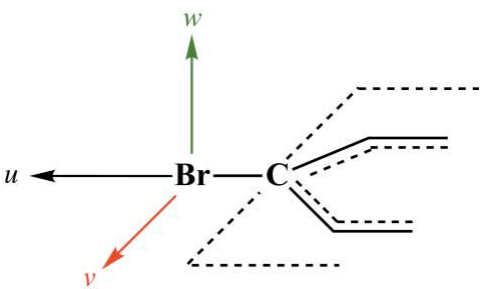

Figure 3

Local coordinate system assigned to the $\mathrm{Br}$ atoms in 5-bromuracil.

possible anisotropy of anomalous scattering in brominated nucleobases, we have mapped the set of the seven experimental spectra onto molecular directions. Principal directions for the anomalous scattering tensors can be inferred from the symmetry of the local environment of the $\mathrm{Br}$ atoms (see Fig. 3) with (i) the direction parallel to the $\mathrm{C}-\mathrm{Br}$ bond (designated u), (ii) the direction perpendicular to the $\mathrm{C}-\mathrm{Br}$ bond and parallel to the plane of the nucleobase ring (designated $\mathbf{v}$ ) and (iii) the direction perpendicular to the nucleobase ring (designated w). For each of the four symmetry-related Br sites $j$ in the unit cell, the anomalous scattering tensors can be expressed by the similarity transformations ${ }^{1}$

$$
\mathbf{f}_{j}^{\prime}=\mathbf{B}_{j} \Delta^{\prime} \mathbf{B}_{j}^{\mathrm{T}}
$$

and

$$
\mathbf{f}_{j}^{\prime \prime}=\mathbf{B} \boldsymbol{\Delta}^{\prime \prime} \mathbf{B}_{j}^{\mathrm{T}},
$$

where $\mathbf{B}_{j}$ are orthogonal matrices whose columns are the components of the vectors defining the principal directions $\mathbf{u}$, $\mathbf{v}$ and $\mathbf{w}$ in whatever orthonormal basis has been chosen, and the matrices $\boldsymbol{\Delta}^{\prime}$ and $\boldsymbol{\Delta}^{\prime \prime}$ are diagonal matrices whose elements denote the principal values along the molecular directions $\mathbf{u}, \mathbf{v}$ and $\mathbf{w}$,

$$
\boldsymbol{\Delta}^{\prime}=\left(\begin{array}{ccc}
f_{\mathrm{u}}^{\prime} & 0 & 0 \\
0 & f_{\mathrm{v}}^{\prime} & 0 \\
0 & 0 & f_{\mathrm{w}}^{\prime}
\end{array}\right)
$$

and

$$
\boldsymbol{\Delta}^{\prime \prime}=\left(\begin{array}{ccc}
f_{\mathrm{u}}^{\prime \prime} & 0 & 0 \\
0 & f_{\mathrm{v}}^{\prime \prime} & 0 \\
0 & 0 & f_{\mathrm{w}}^{\prime \prime}
\end{array}\right) .
$$

Since each experimental spectrum $f_{k}^{\prime}, f_{k}^{\prime \prime}$ is associated with a particular polarization direction $\mathbf{p}_{k}$ in the crystal, the seven polarized spectra were used to solve the linear equations for the unknown principal values $f_{\mathrm{u}}^{\prime}, f_{\mathrm{v}}^{\prime}, f_{\mathrm{w}}^{\prime}$ and $f_{\mathrm{u}}^{\prime \prime}, f_{\mathrm{v}}^{\prime \prime}, f_{\mathrm{w}}^{\prime \prime}$,

$$
f_{k}^{\prime}=\frac{1}{4} \sum_{j=1}^{4} \mathbf{p}_{k}^{\mathrm{T}} \mathbf{f}_{j}^{\prime} \mathbf{p}_{k} \quad k=1, \ldots, 7,
$$

\footnotetext{
$\mathbf{1}^{\mathbf{1}}$ Tensors are denoted in bold type letters. Within the framework of the dipole approximation, only second-rank tensors occur and these are represented by $3 \times 3$ matrices, which are expressed in a crystal Cartesian (orthonormal) basis. The direction of linear polarization of the incident X-ray beam is denoted by the unit vector $\mathbf{p}$, which is also expressed in the orthonormal crystal basis. Thus, our frame of reference is the crystal, not the laboratory, and, from this point of view, changing the crystal orientation actually is a change of the direction of $\mathbf{p}$. See discussion by Bricogne et al. (2005).
} 
and

$$
f_{k}^{\prime \prime}=\frac{1}{4} \sum_{j=1}^{4} \mathbf{p}_{k}^{\mathrm{T}} \mathbf{f}_{j}^{\prime \prime} \mathbf{p}_{k} \quad k=1, \ldots, 7
$$

Both systems of linear equations were solved by singular-value decomposition at every energy point $E$, and the resulting spectra for the principal values are plotted in Fig. 4. These results are in good agreement with earlier data obtained for a $\mathrm{Br}$ attached to a benzene ring (Templeton \& Templeton, 1995; Bricogne et al., 2005.

The $f^{\prime \prime}$ spectra show substantial variations with orientation. In particular, the white line is most pronounced along $\mathbf{u}$ (i.e. the direction parallel to the $\mathrm{C}-\mathrm{Br}$ bond), whereas it completely disappears when the polarization vector of the $\mathrm{X}$-ray beam is perpendicular to the $\mathrm{C}-\mathrm{Br}$ bonds. In the $f^{\prime}$ spectra, a dramatic shift by more than $7 \mathrm{eV}$ in the energy position of the minimum (the so-called inflection point) is observed when the direction of polarization is changed from parallel to perpendicular to the $\mathrm{C}-\mathrm{Br}$ bonds.

The white lines in the X-ray absorption spectra of simple solids have been qualitatively explained as being due to electronic transitions into a high density of final states (Brown et al., 1977). For example, at the $K$-absorption edge, a $1 s$ electron makes a transition into empty final states of ' $p$ ' symmetry, as dictated by the dipole selection rules $(\Delta l= \pm 1)$. By analogy, for molecular compounds, a resonance giving rise to a white line at the $K$ edge can be understood in the one electron picture as the transition of a $1 s$ core electron to an empty antibonding molecular orbital of ' $p$ ' symmetry. In 5bromouracil, the exact site symmetry of the $\mathrm{Br}$ atom is $C_{s}$, but can be approximated by $C_{2 v}$ with the twofold symmetry axis along the $\mathrm{C}-\mathrm{Br}$ bond if only the nearest neighbour atoms are considered. From our experimental data, we observe that the white line is absent for all polarization directions perpendi-

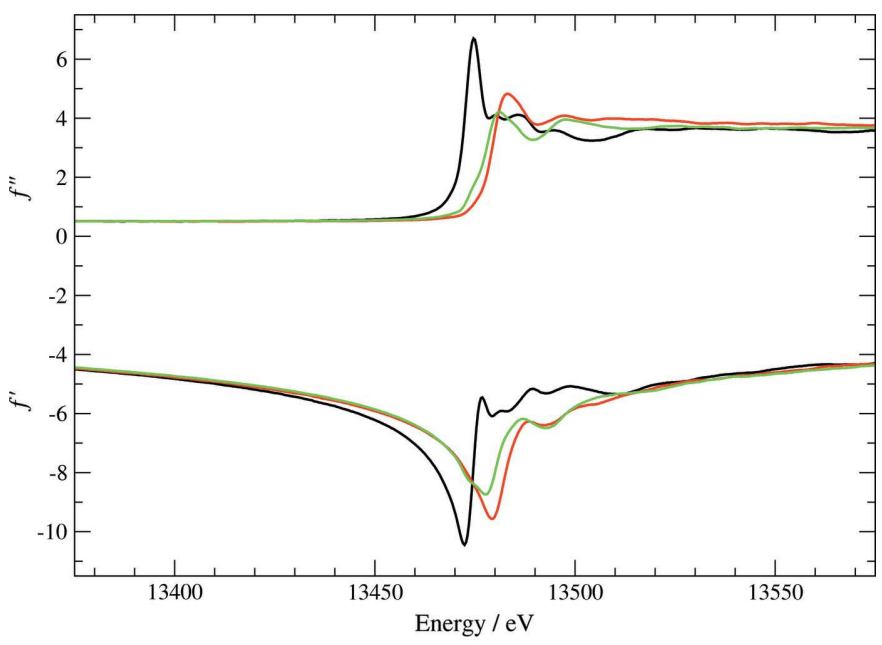

Figure 4

Principal values of the anomalous scattering tensors for $\mathrm{Br}$ in 5bromouracil. Black curves: along direction $\mathbf{u}$ (parallel to the $\mathrm{C}-\mathrm{Br}$ bond). Red curves: along direction $\mathbf{v}$ (perpendicular to the $\mathrm{C}-\mathrm{Br}$ bond and parallel to the plane of the nucleobase ring). Green curves: along direction $\mathbf{w}$ (perpendicular to the nucleobase ring). cular to the $\mathrm{C}-\mathrm{Br}$ bond. We thus conclude that the antibonding orbital involved in the white-line must be of symmetry $A_{1}$ (in $C_{2 v}$ ). In compounds where the $\mathrm{Br}$ is bound to an $s p^{2} \mathrm{C}$ atom, this is the antibonding $\sigma^{*}(p)$ molecular orbital, which is a linear combination of the $\mathrm{C} 2 s p_{z}^{2}$ and $\mathrm{Br} 4 p_{z}$ orbitals, respectively ( $z$ being oriented along the $\mathrm{C}-\mathrm{Br}$ bond). This interpretation is supported by earlier $\mathrm{X}$-ray absorption studies on Br-doped polyacetylene (Oyanagi et al., 1984) and by our observation that the polarized absorption spectra of 5bromouracil are virtually identical to those of other brominesubstituted aromatic compounds (Schiltz et al., unpublished results). We therefore assert that the $f^{\prime}$ and $f^{\prime \prime}$ reference data, here derived for 5-bromouracil, may also serve as standards for other bromine-substituted nucleotides.

\section{Polarization-dependence of anomalous scattering in crystals of brominated DNA}

Since brominated nucleobases are key compounds in SAD/ MAD phasing of nucleic acids, we investigated the possible impact of the anisotropy of anomalous scattering on the experimental phasing of the brominated Z-DNA duplex $\mathrm{d}(\mathrm{CGCG}[\mathrm{BrU}] \mathrm{G})$.

\subsection{Experimental}

The brominated Z-DNA duplex d(CGCG[BrU]G) was produced by chemical synthesis along standard lines. There are two brominated residues per DNA duplex. A single crystal of size $80 \times 200 \times 100 \mu \mathrm{m}$ was mounted on a nylon fibre loop and flash frozen in liquid nitrogen. All measurements were carried out on the SBC-CAT beamline 19ID at the Advanced Photon Source (APS) in Argonne (IL), USA (Rosenbaum et $a l ., 2006)$. The crystal was oriented with the aid of a computercontrolled Kappa goniostat. The crystal symmetry is $P 2_{1} 2_{1} 2_{1}$. This can give rise to differences in the $f^{\prime \prime}$ spectra recorded with

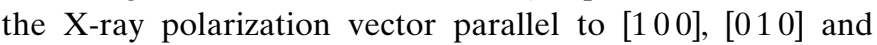
[0 01 1], respectively. X-ray absorption spectra were recorded in fluorescence excitation mode on a single cryo-frozen crystal in three different orientations, i.e. with the polarization vector (I)

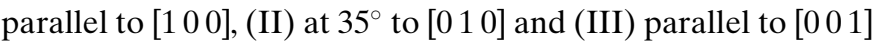
(because of angular restrictions of the crystal goniostat instrument used in these experiments, it was not possible to

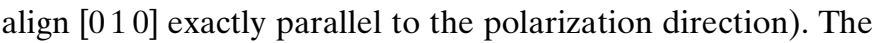
diffraction data were then collected for the same three crystal orientations, in the same order at the single wavelength of $0.9199 \AA$, corresponding to the energy position of the white line $(13.477 \mathrm{keV})$. The data were separately integrated for each set using MOSFLM (Leslie, 1993). Further data processing was carried out using programs of the CCP4 software suite (CCP4, 1994). Details of the data collection and reduction parameters for the three data sets are presented in Table 1 . The $\mathrm{Br}$ sites were located by anomalous-difference Patterson maps. Heavy atom refinement and phasing were carried out along the standard lines using SHARP (Bricogne et al., 2003), followed by density modification using SOLOMON (Abrahams \& Leslie, 1996). The structure was refined with 
Table 1

Data collection and processing statistics for the brominated Z-DNA duplex d(CGCG[BrU]G).

Values in parentheses refer to the outer resolution shell.

\begin{tabular}{|c|c|c|c|}
\hline & Data set (I) & Data set (II) & Data set (III) \\
\hline $\mathrm{X}$-ray polarization direction $(\mathbf{p})$ & Parallel to $\left[\begin{array}{lll}1 & 0 & 0\end{array}\right]$ & At $35^{\circ}$ to $[010]$ & Parallel to $\left[\begin{array}{lll}0 & 0 & 1\end{array}\right]$ \\
\hline X-ray wavelength/photon energy & & $0.9199 \AA ̊ \AA / 13.477 \mathrm{keV}$ & \\
\hline Rotation per image $\left(^{\circ}\right)$ & 1 & 1 & 1 \\
\hline Exposure time per image (s) & 2.2 & 2.2 & 2.2 \\
\hline Total number of images & 145 & 145 & 149 \\
\hline Space group & \multirow{2}{*}{\multicolumn{3}{|c|}{$\begin{array}{l}P 2_{1} 2_{1} 2_{1} \\
17.34 \AA \text { 足, } 32.07 \AA, 44.34 \AA, 90^{\circ}, 90^{\circ}, 90^{\circ}\end{array}$}} \\
\hline Cell parameters & & & \\
\hline Resolution limits $(\AA)$ & $32.1-1.10(1.16-1.10)$ & $30.0-1.10(1.16-1.10)$ & $32.1-1.10(1.16-1.10)$ \\
\hline Number of measured reflections & $40340(1002)$ & 38694 (999) & $42062(1037)$ \\
\hline Number of unique reflections & $8284(443)$ & $9259(603)$ & $8534(496)$ \\
\hline Data completeness $\dagger(\%)$ & $78.8(30.8)$ & $87.5(40.4)$ & $81.0(34.0)$ \\
\hline Anomalous completeness & $75.5(23.1)$ & $77.1(18.5)$ & $74.5(18.8)$ \\
\hline$R_{\text {merge }}$ & $0.053(0.207)$ & $0.061(0.206)$ & $0.046(0.173)$ \\
\hline$R_{\text {meas }} \S$ & $0.065(0.286)$ & $0.075(0.286)$ & $0.056(0.242)$ \\
\hline$R_{\text {meas } 0} \oplus$ & $0.091(0.300)$ & $0.121(0.321)$ & $0.064(0.251)$ \\
\hline SAD phasing power & 1.63 & 1.93 & 1.05 \\
\hline SAD figures of merit $\dagger \dagger$ & 0.44 & 0.45 & 0.29 \\
\hline
\end{tabular}

† For all data sets, the completeness is better than $98 \%$ in all resolution shells up to $1.3 \AA$ resolution. the anomalous completeness is better than $96 \%$ in all resolution shells up to $1.3 \AA$ resolution. $\S$ Redundancy independent (multiplicity-weighted) $R_{\text {merge }}$, keeping Bijvoet pairs separate (i.e. computed in the crystal point group). - Redundancy-independent (multiplicity-weighted) $R_{\text {merge }}$, not keeping Bijvoet pairs separate (i.e. computed in the crystal Laue group). †† After heavy-atom refinement (in SHARP) and before solvent flattening. recorded here are, however, not of the quality of those measured on 5bromouracil. They were measured under experimental conditions closely matching those of a standard MAD experiment, where the absorption spectra are collected in rather short times and thus with limited counting statistics, because they are primarily used to select the optimal wavelengths for performing the subsequent diffraction measurements. Nevertheless, the data clearly show that the white line is completely absent in the spectrum where the polarization direction parallels [001], whereas it is most pronounced when the polarization direction is close to [0 10$]$. For each of the three crystal orientations, structure determination via SAD phasing was attempted with established procedures. Anomalous-difference Patterson maps display significant variations of the signal strengths as a function of the
Table 2

Refinement statistics for the brominated Z-DNA duplex $\mathrm{d}(\mathrm{CGCG}[\mathrm{BrU}] \mathrm{G})$.

\begin{tabular}{ll}
\hline Resolution limits $(\AA)$ & $25.985-1.100$ \\
Number of used reflections & 9673 \\
Percentage observed (completenes) & 96.1648 \\
Percentage of free reflections & 4.7464 \\
Overall $R$ factor & 0.2017 \\
Free $R$ factor & 0.2133 \\
Overall weighted $R$ factor & 0.2017 \\
Free weighted $R$ factor & 0.2133 \\
Overall correlation coefficient & 0.9550 \\
Free correlation coefficient & 0.9567 \\
Cruickshanks DPI for coordinate error & 0.0464 \\
DPI based on free $R$ factor & 0.0425 \\
Overall figure of merit & 0.8787 \\
ML based s.u. of positional parameters & 0.0260 \\
ML based s.u. of thermal parameters & 1.0929 \\
R.m.s.d. bond distance $(\AA)$ & 0.007 \\
R.m.s.d. bond angle $\left(^{\circ}\right)$ & 1.868 \\
\hline
\end{tabular}

reflection data obtained from merging all three orientations using the program REFMAC (Murshudov et al., 1997). Water molecules were located by weighted difference Fourier maps. Anisotropic displacement parameters were refined for all DNA atoms, and hydrogen atoms were included at their idealized positions. The refinement statistics are reported in Table 2. The coordinates of the final structure model have been deposited in the RCSB Protein Data Bank (accession number 2OBZ).

\subsection{Results and discussion}

The absorption spectra display marked variations in the near-edge features (Fig. 5), concurring with the features observed for crystals of 5-bromouracil. The absorption spectra crystal orientation (Fig. 6). Only with the data from the orientations (I) and (II) was it possible to solve the crystal structure, with the data (II) yielding by far the best electron density map (Fig. 7). An inspection of the molecular structure reveals the origin of the strong directional dependence of the anomalous scattering (Fig. 8): all $\mathrm{C}-\mathrm{Br}$ bonds in the unit cell are located in planes almost perpendicular to [001] and preferentially oriented along [0 100$]$.

The data were analysed with great care in order to assess whether X-ray-induced radiation damage at the $\mathrm{Br}$ sites would have caused the failure of phase determination from data set (III) (Ennifar et al., 2002). For all data sets, dose-dependent occupancy decay factors were refined for the $\mathrm{Br}$ atoms (Schiltz

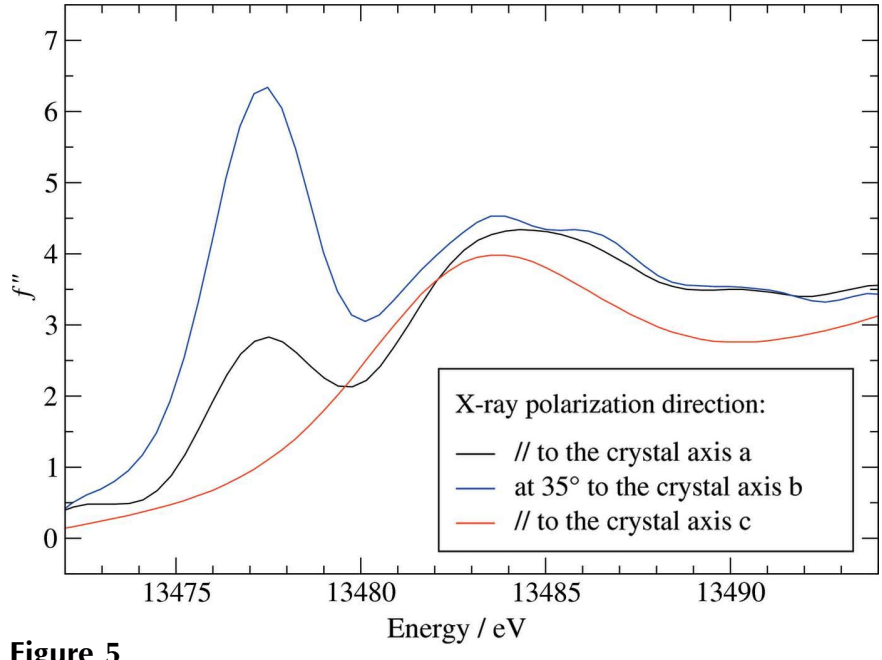

Polarized spectra of the $f^{\prime \prime}$ anomalous scattering factor in the vicinity of the $\mathrm{Br} K$ edge, recorded for three different orientations (see inset) of a single crystal of $\mathrm{d}(\mathrm{CGCG}[\mathrm{BrU}] \mathrm{G})$. 


\section{research papers}

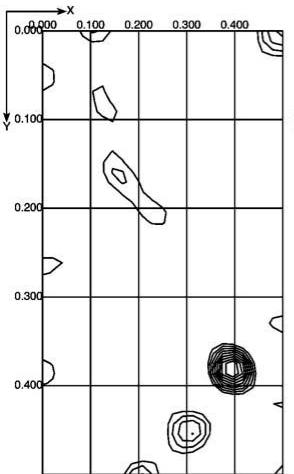

(a)

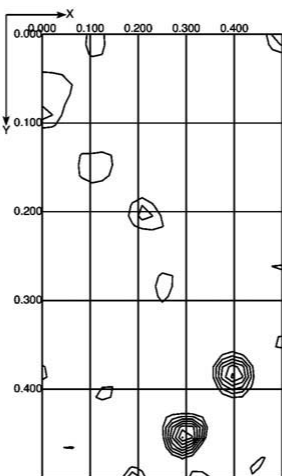

(b)

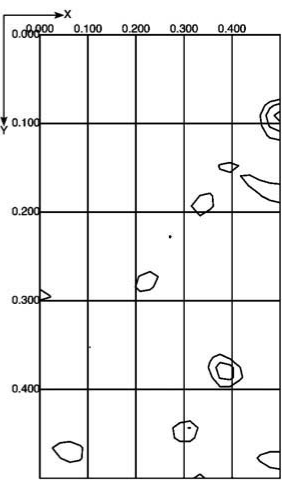

(c)
Figure 6

Anomalous-difference Patterson maps (asymmetric unit of the Harker section $z=1 / 2)$ of $\mathrm{d}(\mathrm{CGCG}[\mathrm{BrU}] \mathrm{G})$. Maps were computed from diffraction data recorded with the X-ray polarization direction $(a)$ parallel to [1 000$],(b)$ at $35^{\circ}$ to [ 010$]$ and $(c)$ parallel [ 00101 . Contours are at intervals of $1 \sigma$ (where $\sigma$ is the root-mean-square of the map density), starting at $2 \sigma$ above the mean density.

et al., 2004) but their final values were very close to zero. Also, for the data set (II), the white line in the absorption spectrum is largest. Had there been significant radiation damage at the $\mathrm{Br}$ sites, one would have expected a continuous decrease in the anomalous signal strength from the first to the second data set (Ennifar et al., 2002). This was not, however, the case. Finally, refinements of the whole Z-DNA duplex structure were independently carried out using each of the three data sets. No significant changes in the occupancies of the $\mathrm{Br}$ atoms were observed. Since the crystals are strongly diffracting, even at high scattering angles, synchrotron data of non-brominated Z-DNA duplex could be collected to $0.55 \AA$ resolution (Sanishvili, unpublished results). Thus, the diffraction data reported here were recorded quickly and with a minimal X-ray dose. We therefore conclude that radiation damage at the $\mathrm{Br}$

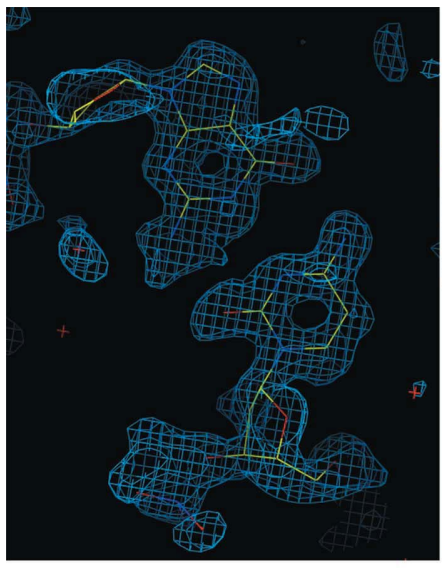

(a)

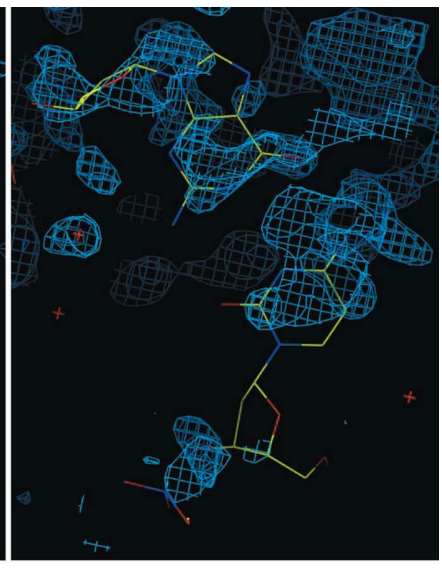

(b)
Figure 7

Electron density maps of $\mathrm{d}(\mathrm{CGCG}[\mathrm{BrU}] \mathrm{G})$ based on SAD phases (calculated using SHARP, followed by density modification using $S O L O M O N)$. The phases were computed from diffraction data recorded with the X-ray polarization direction $(a)$ at $35^{\circ}$ to $[010]$ and $(b)$ parallel to [001]. Both maps show the same region of the electron density (in chicken-wire representation), with the refined structural model of $\mathrm{d}(\mathrm{CGCG}[\mathrm{BrU}] \mathrm{G})$ superimposed.

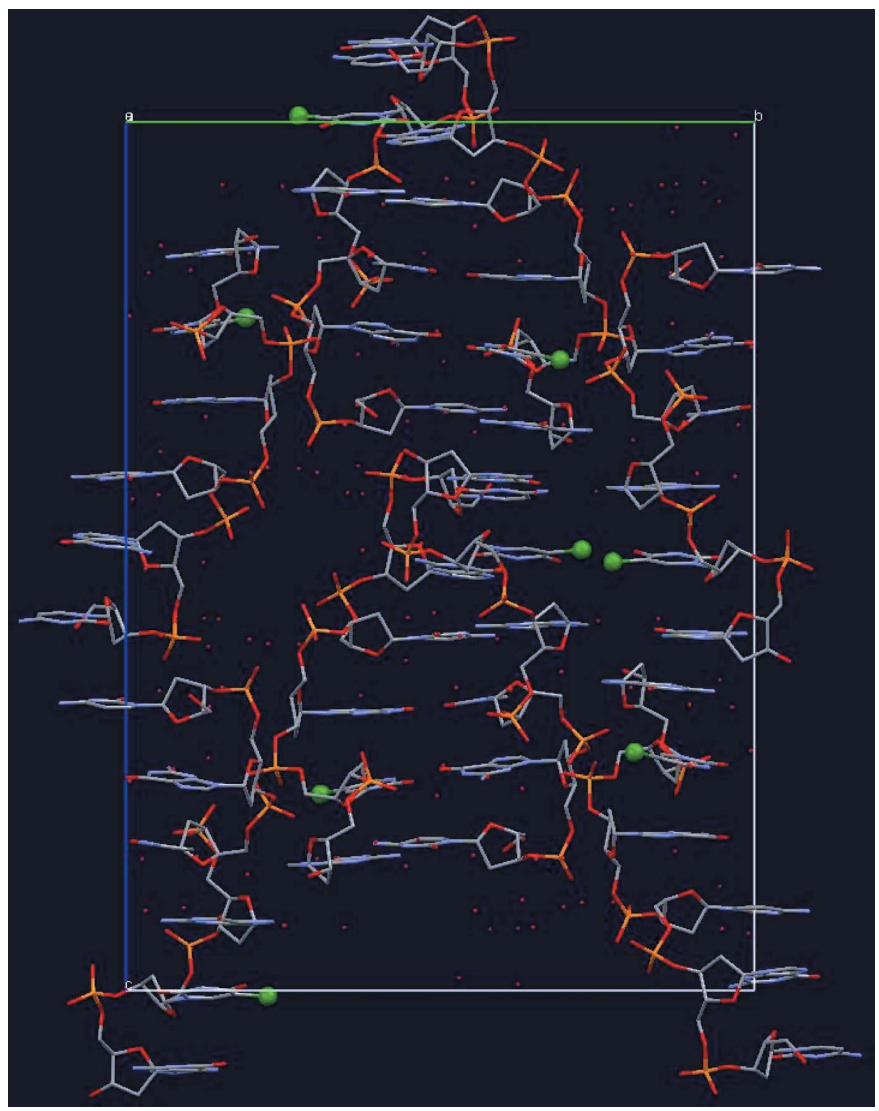

Figure 8

Packing of $\mathrm{d}(\mathrm{CGCG}[\mathrm{BrU}] \mathrm{G})$ molecules viewed along [1 000$]$. The eight $\mathrm{BrU}$ moieties in the unit cell are displayed, with the $\mathrm{Br}$ atoms highlighted as green spheres. Because of the orientation of the helical DNA duplexes in the crystal, all $\mathrm{C}-\mathrm{Br}$ bonds are almost perpendicular to [0 0101$]$.

sites was not an issue and that the observed modulations of the anomalous signal strengths can be exclusively attributed to anisotropic anomalous scattering, as discussed above.

\section{Conclusion}

Our results demonstrate that there is substantial anisotropy of anomalous scattering in the vicinity of the $\mathrm{Br} K$ edge in brominated nucleotides. Thus, when applying SAD phasing near the $K$-absorption edge of brominated DNA or RNA molecules, the correct choice of crystal orientation with respect to the direction of the synchrotron radiation polarization can be as crucial for a successful structure determination as is the correct choice of wavelength. Obviously, selecting the optimal crystal orientation will also be important for improving the quality of phases obtained from MAD experiments. The present study shows furthermore that absorption spectra recorded on randomly oriented single crystals cannot necessarily be transfered from one sample to another. These conclusions are particularly important for brominated nucleic acid structures, since the stacking of bases in the DNA molecules implies that the directions of all $\mathrm{C}-\mathrm{Br}$ bonds in the molecule are confined to more-or-less parallel planes perpendicular to the helix axis. Thus, even if there are 


\section{research papers}

several brominated residues in the molecule, the directions of the $\mathrm{C}-\mathrm{Br}$ bonds are likely to produce a net overall directional dependence of anomalous scattering. Also, in the vast majority of nucleic acid crystal structures, the helix axis coincides with one of the crystal axes (Timsit \& Moras, 1992; Berman, 1997), so that the specific resonant enhancement of the anomalous signal (due to the white line) cannot be exploited along that direction. In fact, the reference data obtained on 5-bromouracil show that the optimum choice of wavelengths in a MAD experiment cannot be made independent from the crystal orientation, as the energies of the extremum values in the $f^{\prime}$ and $f^{\prime \prime}$ spectra depend on the sample orientation. In the context of anomalous scattering experiments on selenated protein crystals of non-cubic symmetry, we have proposed earlier that the optimal crystal orientation can be determined from a series of polarized absorption spectra on differently oriented samples without needing a priori knowledge of the molecular structure (Bricogne et al., 2005). This procedure can also be applied to crystals of brominated nucleic acids, as was shown here. The practical implementation of this method requires, however, the availability of computer-controlled multi-axis goniostats at synchrotron beamlines. Wavelength tunability and stability are nowadays prime objectives in the design of MAD-capable beamlines, and adequate instrumentation for the recording of absorption spectra is considered to be essential. The present study suggests that also multi-axis goniostats should become an indispensable part of the equipment, since anomalous scattering properties can generally depend on both radiation energy and crystal orientation.

We thank Y. G. Gao and A. H. J. Wang for the gift of d(CGCG[BrU]G) crystals. We thank P. Roversi, G. Evans and S. C. Capelli for their contributions during the early stages of this project. We acknowledge financial support from European Commission Grant No. LSHG-CT-2003-503420 within the BIOXHIT project and from Swiss National Science Foundation Grant No. 200021-101519/1. We acknowledge the Advanced Photon Source (APS), the Structural Biology Center CAT, the European Synchrotron Radiation Facility and the Swiss-Norwegian Beamline Consortium for provision of synchrotron radiation facilities and user beamtime. Use of the Advanced Photon Source was supported by the US Department of Energy, Basic Energy Sciences, Office of Science, under contract No. W-31-109-ENG-38.

\section{References}

Abrahams, J. P. \& Leslie, A. G. W. (1996). Acta Cryst. D52, 30-42. Berman, H. M. (1997). Biopolymers, 44, 23-44.

Birkedal, H. (2000). Use of Synchrotron Radiation in Chemical Crystallography. Université de Lausanne, Lausanne, Switzerland.

Bricogne, G., Capelli, S. C., Evans, G., Mitschler, A., Pattison, P., Roversi, P. \& Schiltz, M. (2005). J. Appl. Cryst. 38, 168-182.

Bricogne, G., Vonrhein, C., Flensburg, C., Schiltz, M. \& Paciorek, W. (2003). Acta Cryst. D59, 2023-2030.

Brown, M., Peierls, P. E. \& Stern, E. A. (1977). Phys. Rev. B, 15, 738 744.

Collaborative Computational Project, Number 4 (1994). Acta Cryst. D50, 760-763.

Correll, C. C., Freeborn, B., Moore P. B. \& Steitz, T. A. (1997). J. Biomol. Struct. Dynam. 15, 165-172.

Dmitrienko, V. E. (1983). Acta Cryst. A39, 29-35.

Dmitrienko, V. E., Ishida, K., Kirfel, A. \& Ovchinnikova, E. N. (2005). Acta Cryst. A61, 481-493.

Ennifar, E., Carpentier, P., Ferrer, J.-L., Walter, P. \& Dumas, P. (2002). Acta Cryst. D58, 1262-1268.

Evans, G. \& Pettifer, R. F. (2001). J. Appl. Cryst. 34, 82-86.

Fanchon, E. \& Hendrickson, W. A. (1990). Acta Cryst. A46, 809820.

González, A. (2003). Acta Cryst. D59, 1935-1942.

Hendrickson, W. A. (1991). Science, 254, 51-58.

Hendrickson, W. A. (1999). J. Synchrotron Rad. 6, 845-851.

Hendrickson, W. A., Horton, J. R. \& LeMaster, D. M. (1990). EMBO J. 9, 1665-1672.

Hendrickson, W. A., Smith, J. L., Phizackerley, R. P. \& Merritt, E. A. (1988). Proteins Struct. Funct. Genet. 4, 77-88.

Kirfel, A., Petcov, A. \& Eichhorn, K. (1991). Acta Cryst. A47, 180195.

Leslie, A. G. W. (1993). Proceedings of CCP4 Study Weekend on Data Collection and Processing, pp. 44-51. Daresbury: SERC Daresbury Laboratory.

Murshudov, G. N., Vagin, A. A. \& Dodson, E. J. (1997). Acta Cryst. D53, 240-255.

Ogata, C. M. (1998). Nature Struct. Biol. 5, 638-640.

Oyanagi, H., Tokumoto, M., Ishiguro, T., Shirakawa, H., Nemoto, H., Matsushita, T., Ito, M. \& Kuroda, H. (1984). J. Phys. Soc. Jpn, 53, 4044-4053.

Rosenbaum, G. et al. (2006). J. Synchrotron Rad. 13, 30-45.

Schiltz, M., Dumas, P., Ennifar, E., Flensburg, C., Paciorek, W., Vonrhein, C. \& Bricogne, G. (2004). Acta Cryst. D60, 10241031.

Sternglanz, H. \& Bugg, C. E. (1975). Biochim. Biophys. Acta, 378, 111.

Templeton, D. H. \& Templeton, L. K. (1982). Acta Cryst. A38, 6267.

Templeton, L. K. \& Templeton, D. H. (1988). Acta Cryst. A44, 10451051.

Templeton, L. K. \& Templeton, D. H. (1995). J. Synchrotron Rad. 2, 31-35.

Timsit, Y. \& Moras, D. (1992). Methods in Enzymology, Vol. 211, edited by D. M. J. Lilley \& J. E. Dahlberg, pp. 409-429. London: Academic Press. 